\title{
Utilización y cumplimiento en la unidad de recién nacidos, Hospital de San José
}

José Junco, MD*, Beatriz Haydar MD*, Fabio Espinosa, MD**

\section{Resumen}

Objetivo: describir la utilización y cumplimiento de los criterios de transfusión (TR) de Shannon en la unidad de recién nacidos (RN) del Hospital de San José.

Método: estudio observacional descriptivo, retrospectivo, donde se incluyeron $67 \mathrm{RN}$ prematuros que requirieron TR en el hospital de San José, entre junio 2004 y junio 2005. Se tomó como parámetro más importante la variable dependiente nivel de hematocrito (Ht).

Resultados: el nivel de Ht más frecuente se ubicó en el grupo de 21 a $30 \%$, los promedios de la edad gestacional al nacimiento y del peso promedio fueron 30.4 semanas y $1.305 \mathrm{~g}$. Se cruzó la variable $\mathrm{Ht}$ con el resto que forman parte de los criterios de transfusión de Shannon, encontrándose que el único paciente con nivel de Ht menor de $20 \%$ no cumplió criterio, 36 entre 21 y $30 \%$ cumplieron con el $100 \%$ de los criterios establecidos y 19 entre $31-35 \%$, se transfundieron cumpliendo los criterios. El grupo con nivel mayor de 35\% no tenía criterios de transfusión. Ninguno de los pacientes transfundidos presentó complicaciones. Conclusión: el $\mathbf{8 3 . 5 8 \%}$ de los prematuros que fueron transfundidos cumplieron los criterios establecidos (Shannon), mientras que un $\mathbf{1 6 . 4 2 \%}$ no. Se planteó la pregunta: ¿Implementar los criterios de transfusión de Shannon como protocolo en la unidad de recién nacidos del Hospital de San José reduciría este porcentaje?

Palabras clave: anemia, transfusión, prematuros, hematocrito.

Abreviaturas: TR, transfusión (es); RN, recién nacido; AP, anemia del prematuro; Hb, hemoglobina; Ht, hematocrito.

\section{Introducción}

Este trabajo analiza el número de TR realizadas en los RN pretérmino entre 28 y 34 semanas de edad gestacional nacidos en el Hospital de San José, además de describir la utilización y cumplimiento de los criterios universales establecidos para la TR en prematuros (Shannon).

La importancia de la AP y de las numerosas TR sanguíneas a que se exponen estos pacientes plantean este interrogante: ¿Se podría reducir el número de TR

Fecha recibido: 20 de abril de 2006

Fecha aceptado: 2 de mayo de 2006

Residentes III de pediatría, Fundación Universitaria de Ciencias de la Salud, Hospital de San José.

** Médico pediatra, servicio de pediatría, Hospital de San José. Instructor Asistente Fundación Universitaria de Ciencias de la Salud. que recibe cada paciente, considerando los riesgos que esta terapia conlleva?

Se ha estimado que 38.000 neonatos reciben al año más de 300.000 transfusiones. La tendencia mundial ha sido disminuirlas. Así, en Estados Unidos se pasó de siete TR en los años ochenta a 2.3 en promedio por paciente en los noventa. Se calcula que entre 60 y $80 \%$ de los prematuros de muy bajo peso al nacimiento, reciben por lo menos una TR de sangre durante su hospitalización.

La AP afecta en especial a los menores de 32 semanas de gestación. Se desarrolla entre la tercera y decimosegunda semana de vida y es la forma más frecuente de anemia que se ve en neonatología. Sus características patogénicas la hacen especial y, por ende, su abordaje terapéutico es diferente al de otros tipos de anemia del recién nacido. 


\section{Tabla I. Criterios de transfusión de Shannon*}

Ht entre 31 y $35 \%$ :

- Si recibe $>35 \%$ de oxígeno complementario.

- Si está intubado en CPAP o recibe asistencia mecánica respiratoria con presión media de la vía aérea de 6-8 $\mathrm{cm} \mathrm{H}_{2} 0$.

Ht entre 21 y $30 \%$ :

- Todas las anteriores.

- Si esta recibiendo $<35 \%$ de oxígeno complementario

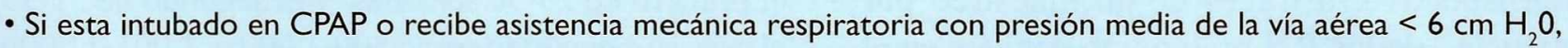

- Aparición de episodios de apnea/bradicardia (>nueve episodios en doce horas o dos episodios en 24 horas que requieran ventilación con bolsa y máscara) estando en tratamiento con dosis terapéuticas de metilxantinas.

- Si presenta taquicardia $(>180 / \mathrm{min})$ o taquipnea $(>80 / \mathrm{min})$ persistente por 24 horas.

- Si el aumento de peso es <10g/día durante cuatro días a pesar de recibir aporte calórico de $100 \mathrm{cal} / \mathrm{K} /$ día.

- Si debe ser intervenido quirúrgicamente.

$\mathrm{Ht}$ menor de $20 \%$ :

- Todas las anteriores

- Si está asintomático, pero con reticulocitos $<1 \%$.

No se transfunden los pacientes por la toma excesiva de muestras para exámenes de laboratorio o con solo bajo nivel de $\mathrm{Ht}$.

*Tomado de: Shannon KM, Keith JF III, Mentzer WC, Recombinant human erythropoietin stimulates erythropoiesis and reduces erythrocyte transfusions in very low birth weight preterm infants. Pediatrics, 1995;95: I-8.

Se define como la disminución fisiológica y en ocasiones extrema de los niveles de $\mathrm{Hb}$ y $\mathrm{Ht}$ entre las seis y doce semanas de vida extrauterina; la intensidad de la caída de $\mathrm{Hb}$ es directamente proporcional al grado de prematurez y afecta sobretodo a los neonatos pretérmino con peso menor de 1.500 $\mathrm{g}$ al nacer.

La necesidad de TR en los $\mathrm{RN}$ prematuros se basa en los niveles de Ht y en ciertas condiciones clínicas, las cuales se establecen con precisión en los criterios de Shannon; estos fueron desarrollados como parte de las guías clínicas utilizadas para el estudio Eritropoyetina recombinante humana, estimula la eritropoyesis y disminuye el riesgo de transfusiones en recién nacidos preterminos de muy bajo peso, publicado en 1995 y de ahí en adelante se han aplicado como protocolo para diferentes hospitales, con algunas variaciones de acuerdo con cada centro (Tabla 1).
Los criterios de Shannon se basan en condiciones patológicas particulares de los neonatos como son ventilación mecánica y requerimientos de oxígeno. Aquellos con soporte ventilatorio alto y corto circuito intrapulmonar aumentado o con enfermedad de espacio muerto, requerirán niveles de $\mathrm{Hb}$ mayores para que no exista compromiso de la oxigenación tisular con todas sus consecuencias.

Además, una disminución en los niveles de $\mathrm{Hb}$ - $\mathrm{Ht}$ conlleva a un aumento en el gasto cardíaco reflejado sobre todo en la frecuencia cardíaca. Con niveles de $\mathrm{Hb}$ disminuidos, su curva de disociación se desplaza hacia la derecha, lo que conlleva a una menor entrega de oxígeno al miocárdico, hipercapnia, taquipnea secundaria y mayor riesgo de apneas. Esto llevaría a un aumento en el gasto calórico y a una inadecuada ganancia de peso. 
Es importante valorar la respuesta medular ante niveles disminuidos de $\mathrm{Hb}$ a través de los niveles de reticulocitos, que son los precursores eritrocitarios.

\section{Métodos}

Se realizó un estudio observacional descriptivo, retrospectivo, a través de un muestreo por conveniencia, de los RN pretérmino nacidos en el Hospital de San José entre junio de 2004 y junio de 2005, donde se incluyeron prematuros de 26 a 34 semanas de edad gestacional transfundidos en esta unidad, y se excluyeron pacientes con enfermedad hemolítica de cualquier tipo, exanguineotransfusiones, cardiopatías congénitas e intervenciones quirúrgicas. Se revisaron 176 historias clínicas de las cuales se tomaron 67 de pacientes que fueron transfundidos. Se tabularon los datos mediante un instrumento diseñado para tal fin en base de datos Excel y el análisis estadístico se realizo mediante el paquete STATA 7.0.

\section{Resultados}

Para este estudio se revisaron 176 historias clínicas de los RN prematuros que ingresaron o nacieron en la unidad de recién nacidos del Hospital de San José en el periodo comprendido entre junio de 2004 y junio de 2005, de estas se tomaron 67 historias que cumplieron los criterios para ser incluidas en el estudio. La categorización de las variables utilizadas se realizó con base en grupos así: días de vida, en grupos de diez días; edad gestacional al nacimiento, que se dividió en dos grupos, el primero de 26-30 semanas y el segundo de 31-35; el peso se categórizo como 500-1.000 g, 1.001-1.500g, $1.501-2.000$ y de 2.001 a $2.500 \mathrm{~g}$. Con respecto a los diagnósticos, los más frecuentes fueron: anemia, hemorragia, enfermedad de membrana hialina, síndrome de disfunción orgánica múltiple y sepsis (Tabla 2).

\section{Análisis}

De los pacientes estudiados, 21 fueron de sexo femenino correspondiente a un $31.3 \%$ del total y 46 masculino para un $68.7 \%$. La edad media de transfusión fue 22.6 días, mediana de 18 con un máximo de 77 y un mínimo de un día; la edad gestacional media al nacimiento fue 30.4 semanas, con una mediana de 30, una máxima de 35 y mínima de 26 semanas; la media para el peso fue $1.305,4 \mathrm{~g}$ y una mediana de $1.306 \mathrm{~g}$, con un límite máximo de 2.200 g y uno mínimo de $700 \mathrm{~g}$.

\begin{tabular}{|c|c|c|}
\hline \multicolumn{4}{|c|}{ Tabla 2. Resultados respecto a sexo, diagnóstico y Ht } \\
\hline SEXO & FRECUENCIA & $\%$ \\
\hline Femenino & 21 & 31.3 \\
\hline Masculino & 46 & 68.7 \\
\hline Total & 67 & 100.0 \\
\hline DIAGNÓsTICO & 49 & 73.1 \\
\hline Anemia & 4 & 6 \\
\hline Hemorragia & 8 & 11.9 \\
\hline Enfermedad de membrana hialina & 2 & 3 \\
\hline Disfusión orgánica & 4 & 6 \\
\hline Sepsis & 67 & 100.0 \\
\hline Total & & 1.5 \\
\hline Ht & 1 & 53.7 \\
\hline$<20$ & 36 & 29.9 \\
\hline $21-30$ & 20 & 14.9 \\
\hline $31-35$ & 10 & 100.0
\end{tabular}




\section{Tabla 3. Resultados con relación a Ht}

\begin{tabular}{|c|c|c|c|c|c|c|c|c|c|c|c|}
\hline Nivel de $\mathrm{Ht}$ & $\begin{array}{l}\text { O2 } \\
\text { supl. } \\
>35 \%\end{array}$ & $\begin{array}{l}\text { VM-CPAP } \\
\text { PMVA 6-8 }\end{array}$ & $\begin{array}{l}\mathrm{FlO} 2 \\
<35 \%\end{array}$ & $\begin{array}{l}\text { CPAP } \\
\text { PMVA } \\
<6\end{array}$ & Apnea & $\begin{array}{l}\text { Taquicardia } \\
\text { Taquipnea }\end{array}$ & $\begin{array}{l}\text { Ganó } \\
\text { peso }\end{array}$ & Cirugía & $\begin{array}{l}\text { Sang } \\
\text { activo }\end{array}$ & $\begin{array}{l}\text { Total } \\
\text { ptes }\end{array}$ & $\%$ \\
\hline$<20 \%$ & 0 & 0 & 0 & 0 & 0 & 0 & 0 & 0 & 0 & 1 & $0 \%$ \\
\hline $21-30 \%$ & 14 & 9 & 19 & 0 & 0 & 3 & 10 & 0 & 0 & 36 & $100 \%$ \\
\hline $31-35 \%$ & 10 & 9 & 0 & 0 & 1 & 0 & 0 & 0 & 0 & 20 & $95 \%$ \\
\hline$>35 \%$ & 7 & 6 & 3 & 0 & 0 & 0 & 5 & 0 & 3 & 10 & $33 \%$ \\
\hline
\end{tabular}

Utilizando como parámetro universal la variable dependiente nivel de Ht se cruzó con el resto de variables independientes involucradas, las cuales forman parte de los criterios de transfusión de Shannon. Se observa que para el nivel de $\mathrm{Ht}<20 \%$, el único paciente de este grupo fue transfundido $\sin$ cumplir con ninguno de los criterios. Entre aquellos con nivel de Ht de $21-30 \%$ se cumplieron un total de 55 criterios y el $100 \%$ de los transfundidos cumplió los criterios (Gráfica 1).

Con nivel de $\mathrm{Ht}$ de $31-35 \%$ fueron transfundidos 20 pacientes, cumpliendo un total de 19 cri- terios. Uno presentó apneas, pero este criterio no se encuentra establecido para niveles de Ht mayores de 30 , por consiguiente de los 20 pacientes transfundidos, el 95\% (19), cumplió con los criterios, mientras que uno no debió ser transfundido con este nivel de Ht.

Entre los pacientes con niveles de Ht mayores de $35 \%$, se encontraron tres con sangrado activo (pulmonar), los cuales se decidieron transfundir por inestabilidad hemodinámica, aunque este nivel de Ht no se encuentra dentro de los criterios de Shannon (Tabla 3).

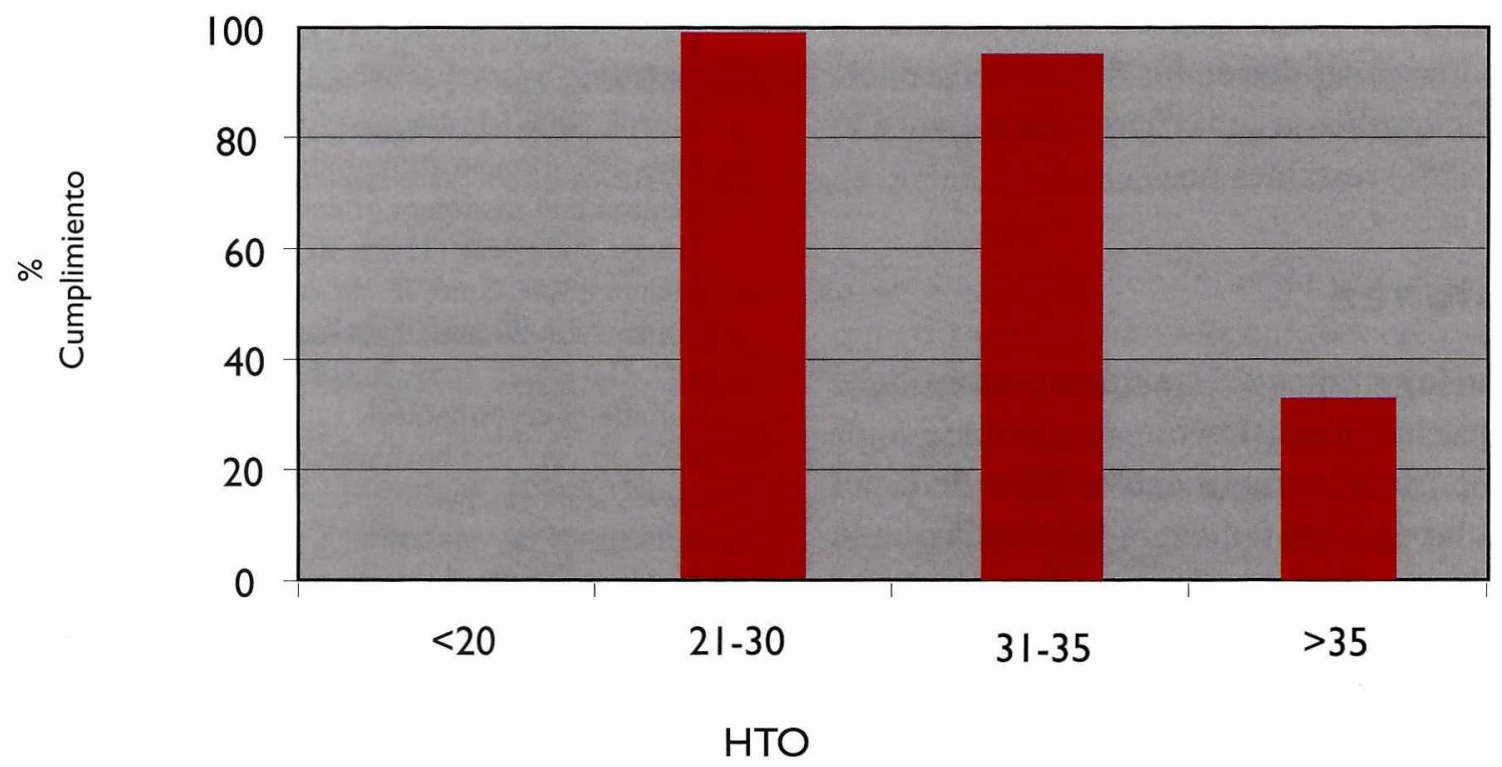

Grafica I. Distribución porcentual de cumplimiento de criterios de acuerdo con el nivel de $\mathrm{Ht}$. 


\section{Discusión}

La AP es una de las complicaciones más frecuentes que se presenta en los pacientes menores de 1.500 gramos de peso al nacer y se ha estimado que 38.000 neonatos reciben más de 300.000 transfusiones al año. La tendencia mundial ha sido a disminuir este último número, lo cual se refleja en las unidades de mayor complejidad al pasar de siete transfusiones en los años ochenta a 2.3 en promedio por paciente en los noventa.

Con este estudio queremos comprobar la utilización adecuada de los criterios de transfusión mundialmente utilizados (Shannon), en la unidad de recién nacidos del Hospital de San José. De los 67 pacientes incluidos, el nivel de Ht con el que más se transfundió fue entre $21-30 \%$ (36 pacientes), que corresponden al 53.73\%. Según los niveles de Ht obtenidos, aquellos con niveles menores de $20 \%$ se transfundieron sin cumplir criterios, entre 21 y $30 \%$ cumplieron el $100 \%$ de los criterios establecidos para este grupo, y de los que cursaron con niveles de Ht entre $31-35 \%$, el 95\% transfundidos cumplieron los criterios. De aquellos con $\mathrm{Ht}$ mayor de $35 \%$, se transfundieron diez pacientes, tres de ellos por inestabilidad hemodinámica y sangrado activo pulmonar, factores que no están incluidos como criterios. De los 67 pacientes transfundidos en los diferentes grupos, $56(83.58 \%)$ cumplieron con criterios de transfusión y los $11(16.42 \%)$ restantes no.

\section{Conclusiones}

$\mathrm{Al}$ valorar los riesgos de las transfusiones debe recordarse que la administración de glóbulos rojos genera un importante y significativo freno de la actividad medular, que profundizará la anemia que se produce por lo regular entre el segundo y cuarto mes de vida en todo RN prematuro. Esta mayor anemia fisiológica se relacionará con un deterioro de la curva pondoestatural, desarrollo potencial de apneas, eventual necesidad de una nueva transfusión de glóbulos rojos, hipervolemia, insuficiencia cardíaca, edema pulmonar, displasia broncopulmonar y mayor riesgo de transmisión de infecciones. La reacción injerto versus huésped, que es más frecuente entre individuos genéticamente cercanos, no hace recomendable la donación de sangre de parientes cercanos al RN. Con frecuencia no se utilizan los criterios de Shannon como protocolo para transfusión en los prematuros, pero si unificamos y nos guiamos en forma estricta por ellos, podríamos reducir al mínimo las transfusiones innecesarias.

\section{Lecturas recomendadas}

- Alkalay AL, Galvis S, Ferry DA, Simmons CF, Krueger RC Jr. Hemodynamic changes in anemic premature infants: are we allowing the hematocrits to fall too low? Pediatrics. 2003 Oct;112(4):838-45.

- Andersen C. Critical haemoglobin thresholds in premature infants. Arch Dis Child Fetal Neonatal Ed. 2001 May;84(3): F146-8

- Becerra M ¿Es Posible Evitar las Transfusiones de Sangre en la Anemia del Prematuro? Red Hospital Clínico Universidad de Chile. Programa de Medicina y Cirugía sin Transfusión Sanguínea [seriada en Internet] 2004 (7) [citado 2006 Sep 12]. Disponible en: http://www.redclinica. cl/pmcsts/articulos/237/05ANEMIAPREMATURO.pdf

- Martínez, JL. Anemia del prematuro. Estrategias terapéuticas. Revista Médica de Clínica Las Condes [seriada en Internet] 1999 Abr [citado 2006 Sep 12];10(1):[aprox. 6 p.]. Disponible en http://www.clc.cl/area_academica/ Revista\%20Medica\%20Abril\%201999/articulo004.htm.

- Ohls RK. The use of erythropoietin in neonates. Clin Perinatol. 2000 Sep;27(3):681-96. Review.

- Ohls RK. Human recombinant erythropoietin in the prevention and treatment of anemia of prematurity. Paediatr Drugs. 2002;4(2):111-21. Review.

- Shannon KM, Keith JF 3rd, Mentzer WC, Ehrenkranz RA, Brown MS, Widness JA, Gleason CA, Bifano EM, Millard DD, Davis CB, et al. Recombinant human erythropoietin stimulates erythropoiesis and reduces erythrocyte transfusions in very low birth weight preterm infants. Pediatrics. 1995 Jan;95(1):1-8.

- Shannon KM, Mentzer WC, Abels RI, Freeman P, Newton N, Thompson D, Sniderman S, Ballard R, Phibbs RH. Recombinant human erythropoietin in the anemia of prematurity: results of a placebo-controlled pilot study. J Pediatr. 1991 Jun;118(6):949-55.

- Widness JA. Pathophysiology, diagnosis and prevention of neonatal anemia. Neoreviews. 2000 Apr;1:e61-e80. 